\title{
A PRELIMINARY REPORT UPON THE OXIDATION OF LIN- SEED OIL.
}

BY FREDERICK I, DUNLAP AKD FRANCIS D. SHEXK.

Receired May 21, 2903.

The question of the phenomenon of the drying of linseed oil is one of great interest, but while more or less work has been clone on this subject, in order to elucidate the chemical changes taking place during the drying of linseed as well as other drying oils, yet our knowledge of the subject is far from complete. We undertook this work with the hope of being able to throw more light on this question, but the fact that we are unable to further carry on this work in conjunction, makes it necessary that this report should be considered merely in the light of a preliminary one, and it is hoped that later, one of us may be able to fill in the gaps that are lacking in the experimental part of this report.

The first work of importance on the subject of the chemistry of drying oils, with reference to the action of air on them, is in the classic work of Mulder, ${ }^{1}$ although previous to Mulder, Unverdorben ${ }^{2}$ and Leuchs ${ }^{3}$ had made a brief study of the action of air on linseed oil, but their results were not such as to lead to any definite conclusions.

Mulder's work on the chemistry of the drying oils, was published in German in I867, and included in it the results of his researches on the action of air on linseed oil as well as on the free acids of linseed oil. At the time when Mulder carried on his researches, the knowledge of the constitution of these oils was very incomplete, nor was much known about them until the publication of the beautiful researches of Hazura and others in the Monatshefte. Mulder came to the conclusion that the liquid acids in linseed oil contained besides oleic acid, an acid of the composition $\mathrm{C}_{10} \mathrm{H}_{28} \mathrm{O}_{2}$ (leinölsäure). On oxidation of linseed oil by means of air, Nulder held that the glycerin was completely oxidized, that the free fatty acids and the greater part of the oleic acid remained unchanged, and that the acid $\mathrm{C}_{1 ;} \mathrm{H}_{28} \mathrm{O}_{2}$ at first formed the acid $\mathrm{C}_{1 ;} \mathrm{H}_{26} \mathrm{O}_{5}$ (linoxysäure) and finally as an end-product of the oxidation, the neutral linoxyn $\mathrm{C}_{32} \mathrm{H}_{54} \mathrm{O}_{11}$.

1 "Chemie der austrocknenden Oele." Translated from the Dutch by J. Miller. Berlin. 1867 . Julius Springer.

2 Schweigger's Jour., 17, 245.

${ }^{3}$ Gmelin-Kraut, 7, 1231 .

4,$216 ; 8,147,156,260 ; 9,180,195,475$. 
Hazura ${ }^{1}$ has shown as the results of his study of the action of potassium permanganate on an alkaline solution of the liquid acids of linseed oil, that linseed oil contains the glycerides of the following acids: Oleic, $\mathrm{C}_{18} \mathrm{H}_{34} \mathrm{O}_{2}$; Linolic, $\mathrm{C}_{18} \mathrm{H}_{32} \mathrm{O}_{2} ;$ Linolenic, $\mathrm{C}_{19} \mathrm{H}_{30} \mathrm{O}_{2}$; Isolinolenic, $\mathrm{C}_{18} \mathrm{H}_{30} \mathrm{O}_{2}$. It was impossible that Mulder should have been able to come to any correct conclusion on the oxidation of linseed oil by means of air, for from the work of Hazura we can see that Mulder was dealing with a more complicated product than he was aware of, and that, ${ }^{2}$ for example, his linoxy acid, $\mathrm{C}_{18} \mathrm{H}_{28} \mathrm{O}_{5}$, obtained from the acid $\mathrm{C}_{18} \mathrm{H}_{28} \mathrm{O}_{2}$, could not have been the simple product that Mulder took it to be, but must have been a mixture of products, which furthermore could not have contained an acid of the chain $\mathrm{C}_{16}$, but must have been those of the higher chain $\mathrm{C}_{18}$.

Bauer and Hazura ${ }^{3}$ studied the phenomenon of the drying of oils, and have clearly shown that the results obtained by Mulder on his linoxy acid are unreliable. So far as the action of air is concerned, Bauer and Hazura studied its action on the free acids of linseed oil, on the barium and lead salts, and on the linseed oil itself. In brief, their conclusions are as follows: The oleic acid undergoes no change whatsoever; the more linolenic acid there is in the oil, the more rapidly it dries; the oxidation consists not only in the saturation of the free valencies with oxygen, but also in the formation of compounds containing hydroxyl groups; the acids dry, on long exposure to the air, with the formation of anhydrides, but that they were unable to say whether such anhydrides were lactonic in nature or formed from 2 molecules of the oxyacids; and finally, that there is no difference between the drying of the free acids and their salts.

For some not very well-defined reason, Bauer and Hazura concluded that as they had shown clearly the constitution of the unsaturated acids in linseed oil, it appeared "unnecessary to isolate the individual compounds from the oxidation product obtained from the acids of linseed oil by the action of air upon them; it is sufficient to show in what way the oxidation takes place." In this, we can not agree with Bauer and Hazura that all that is necessary is to show in what way the oxidation takes place, unless

1 Monatsh. Chem., 9, IgI (1888).

2 Ibrd, , 9, 460 ( 5888$)$.

3 Ibid, p. 459, et seq. 
in so doing, it is perfectly evident what the final products of such an oxidation may be. In order to elucidate the chemical changes taking place when linseed and other oils dry, either the individual oxidation products must be isolated and analyzed, or else, if this prove impracticable, results must be obtained from some other but less direct route. None of the experimental results of Bauer and Hazura can be interpreted to mean that the oxidation by air gives rise to the same products as are obtained by the action of potassium permanganate on an alkaline solution of the linseed oil acids. Nor are the conclusions of Bauer and Hazura as given above, namely, with reference to the formation of hydroxy acids and anhydricles, etc., given with sufficient definiteness to help us to anything like a clear understanding of the question. We have spent considerable time in attempts to isolate some of the hydroxy acids described by Hazura ${ }^{1}$ as resulting from the action of potassium permanganate on the unsaturated acids of linseed oil, but, in the product obtained from the action of air on linseed oil, we have been unable to obtain a trace of any product resembling those obtained by Hazura with potassium permanganate, nor is there any a priori reason for believing that the oxidation would follow the same lines. We judge from the context of the article of Bauer and Hazura that they wish to convey the iclea that air and potassium permanganate produce identical results in the oxidation in question, otherwise we are at a loss to understand why they deem it unnecessary to isolate the oxidation products, but think it sufficient to dismiss the question with a few conclusions quoted at the beginning of this paper.

In I893, Fahrion ${ }^{2}$ published an article on the oxidation of oils by means of air, in which work he gives the results of some oxidation products which he isolated and subjected to analysis. $\mathrm{He}$ oxidized linseed oil, for example, by saturating chamois skin with the oil and allowing the skin so treated to hang in the air until the oxidation was finished. But Fahrion himself shows that he had not obtained pure products, but only mixtures of various oxy-acids.

The above references to the literature give, so far as we have found, the results bearing directly on the chemical changes taking place during the drying of linseed oil.

1 Monalsh. Chem., 9, igr (1888).

: Chem. Zig., 17 (2), 1849 (1893). 
We have attacked the problem in two ways, first, the oxidation of linseed oil when dissolved in ligroin; and second, when dissolved in benzene. A reference to the action of air on linseed oil, when dissolved in benzene, occurs in Allen's "Commercial Organic Analysis." 1 Whether this work is abstracted from a published article or is the result of some experiments carried out by Allen himself, we cannot say, but we have found no trace of any suggestion regarding the oxidation of linseed oil in benzene solution other than that given by Allen. The few lines devoted by Allen to this subject, do not contain any results which cast any light on the chemical side of this phenomenon of oxidation.

The serious objection to the oxidation of linseed oil by spreading it in thin layers on metal or glass plates, and then exposing them to the air, either at ordinary or elevated temperatures, is that it is a process that may take weeks or even months of exposure in order to bring about thorough oxidation, and even then it is a serious question whether or not the oxidation has become complete. As soon as the superficial layer of the oil becomes coated with a layer or film of the oxidized product, the oil lying below this film, and which is unacted upon by the air, is more or less effectively removed from the action of the air by the film on the surface. This latter objection is also applicable to Fahrion's scheme of oxidizing linseed oil which has been exposed to the air on chamois skin.

By dissolving the linseed oil in a suitable solvent, and then aspirating air through it, the above objections are readily overcome. Low-boiling ligroin and benzene serve admirably for this purpose.

The sample of linseed oils used for the experimental part of this work, was a sample of pure boiled oil containing lead and manganese as driers. The iodine number, acid value, etc., of this oil, are given at the beginning of the table later on in this article.

To determine the rate of oxidation, 100 grams of the boiled oil were dissolved in $1,000 \mathrm{cc}$. of ligroin (boiling-point below $90^{\circ}$ ) and air drawn through the solution. This was started November Ioth. After forty-eight hours, the oxidation product that had separated was filtered off, thoroughly macerated with ligroin, then

1 Third edition, Vol. II, Part I, p. 152. 
washed several times with a lower boiling product to remove any of the original linseed oil mechanically held. After drying first in air then in acuo over sulphuric acid, a sharp odor was given off similar to that of hydrochloric acid, only stronger, and resembled the odor of acrolein. This solid oxidation product weighed I7.5 grams. Air was again drawn through the ligroin solution and after forty-eight hours more, a second batch was filtered off. This also had the sharp odor noted above. This lot weighed 24 grams. A third lot was taken out November I Gth ; weight, 25 grams. It did not have the sharp odor-was white and almost transparent. The fourth and last batch was filtered off four days later; it weighed about I 5 grams and looked the same as the third lot. Air was again drawn through the solution until November 24 th, and as no product had separated since the last filtering, it was sufficient evidence that the oxidation was complete November 20 th or before. The total weight of products was 81.5 grams. The remaining solution was clear but fluorescent while cold, and upon distilling off the ligroin in iacuo, the fluorescence disappeared and a precipitate formed. Weight of final product was 20 grams. The ligroin that distilled off had a very sharp penetrating odor like that previously mentioned in connection with the first oxidation products. After standing for a couple of weeks, the sharp odor entirely disappeared from the ligroin. About 5 grams of the last product was solid, the rest a deep yellow oil. The first oxidation products that separated were darker in color than those separated later. During the aspirating of air through the ligroin solution of the linseed oil, the ligroin lost by evaporation was replaced by fresh solvent"so that the volume remained practically constant.

Since the oxidized oil seemed to form two distinct products, so far as color was concerned, an experiment was run to determine whether there was any special difference between them. This was started in the same way as the above experiment, but the oxidation product was allowed to collect until the oxidation was complete. A mechanical separation was then made of these two layers. The dark layer, called A, and the light layer, called B, were each subjected to the method of washing previously mentioned. Each layer weighed approximately 60 grams. They were then sub- 
jected to repeated extractions with boiling ether until no residue remained on evaporating the solvent. The ether extract from $A$ $\left(A_{1}\right)$ weighed 20 grams, while that from $B\left(B_{1}\right)$ weighed 30 . As will be seen from the following table, there is a slight difference in the iodine numbers of the ether-soluble and the ether-insoluble portions of the product $A$. The part $A$ carried more of the manganese of the dryer than the part $B$.

The ether insoluble of $B$ was extracted several times with large quantities of boiling chloroform. Out of $3 \mathrm{I}$ grams taken, only 28 grams were dissolved by the chloroform; this, after evaporating off the solvent, was of a reddish brown color, but gradually turned white on standing. The part insoluble in chloroform was a grayish white product of a rubbery consistency. According to $\mathrm{Mulder}^{1}$ the oxidation product is dissolved by a mixture of alcohol and chloroform, but the products we obtained were not, if conclusions can be drawn from the following experiment. Four-tenths of a gram of the ether-insoluble portion was heated to boiling for sixty hours in a mixture of $400 \mathrm{cc}$. of alcohol and chloroform, one part of the former to four of the latter. At the end of that time a considerable portion was still undissolved. When heated with alcohol and chloroform, the product does swell, but the swelling is due to the chloroform alone, not to the alcohol. It also swells slightly when heated with ether and can be easily ground to a fine powder after the ether is poured off.

On December $5^{\text {th }}$ another sample of 100 grams of the oil was oxidized as the above, but benzene was used instead of ligroin. At first, no product separated as from the ligroin solution. At the end of eighteen days the oil had an iodine number of 37 ; by January $15^{\text {th }}$ the iodine number was 24.9. By February Ist a solid product had separated out; this was filtered off and washed several times with benzene. The dry product, called $\mathrm{C}$, weighed 18 grams. Air was again drawn through the remaining oil for a month more. but nothing further separated. After filtering off the solid product, the oil had an iodine number of 35 , and the lowest we were able to get it after that was $3 \mathrm{I}$. Twenty-two per cent. of the above solid product was soluble in ether, which shows that the product may differ to a certain extent from that separating from the

1 “Chemie der Austrocknenden Oele." p. 98. 
ligroin, as the ether-soluble part of the product separating from ligroin was nearly 50 per cent. of the total solid.

In determining the iodine number, the various samples had to be heated to the temperature of the water-bath in order to effect solution (except in the case of the ether-soluble). When this had been accomplished, Wiji's solution of $\mathrm{ICl}$ in glacial acetic acid was added and allowed to stand for lengths of time varying from fifteen minutes to nearly an hour. In general, the iodine absorption was found to be complete in twenty-five to thirty-five minutes, $i$. e., for a shorter time the iodine absorption was low and increased to a constant when allowed to stand for the period just indicated.

The dissolving of the oxidation product in glacial acetic acid, by means of heat, produced a solution of slightly reddish color. Where, for example, the product was soluble on long shaking with glacial acetic acid at ordinary temperatures, as were the ethersoluble products $A_{1}, B_{1}$ and $C_{1}$, the iodine absorption was found to be less than when such solution was effected by means of heat. For the ether-soluble products $A_{1}, B_{1}$ and $C_{1}$, solution was effected by means of automatic shaking for periods varying from three to ten hours, and in the following table the iodine numbers given represent the results of solution produced without the aid of heat.

$A_{2}$ and $B_{2}$ were nearly all soluble in cold glacial acetic acid and it is highly probable that they are completely soluble if sufficient acid is used, but we were unable to carry out this work for lack of time.

$A_{1}, B_{1}$ and $C_{1}$ were all thick, viscous, yellowish oils, which on long standing in the air grew more viscous, becoming coated with a film like that forming on linseed oil itself. $A_{2}, B_{2}$ and $C_{2}$ were of a leathery or gutta percha-like consistency.

In determining the iodine numbers, Wiji's method was used throughout, as Hübl's method could not be used, for glacial acetic acid was found to be the only satisfactory solvent for these products.

The following table gives some of the results obtained from the various oxidation products. 


\begin{tabular}{|c|c|c|c|c|}
\hline Substance. & $\begin{array}{l}\text { Iodine } \\
\text { number. }\end{array}$ & $\begin{array}{c}\text { Acid value of } \\
\text { potassium } \\
\text { hydroxide. } \\
\text { Milligtams. }\end{array}$ & $\begin{array}{l}\text { Saponification } \\
\text { value of } \\
\text { potassium } \\
\text { hydroxide. } \\
\text { Milligrams. }\end{array}$ & $\begin{array}{l}\text { Ether } \\
\text { value. }\end{array}$ \\
\hline \multirow{2}{*}{ Original oil .. } & $\{176.6$ & 4.00 & 183.0 & 177.8 \\
\hline & $\{176.9$ & $4 . \infty$ & I 80.6 & $\cdots$ \\
\hline \multirow[b]{2}{*}{ A } & $44.8(?)$ & $\cdots$ & $\cdots$ & $\cdots$ \\
\hline & 54.2 & $\cdots$ & $\cdots$ & $\cdots$ \\
\hline \multirow[b]{2}{*}{$B \ldots \ldots$} & 26.7 & $\cdots$ & $\cdots \cdot$ & $\cdots$ \\
\hline & 28.5 & $\cdots$ & $\cdots$ & $\cdots$ \\
\hline \multirow{2}{*}{ 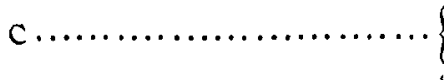 } & $\{22.0$ & $\cdots$ & $\cdots$ & $\cdots \cdot$ \\
\hline & 22.7 & $\cdots$ & $\cdots$ & $\cdots$ \\
\hline \multirow{2}{*}{$A_{1}$, soluble in ether $\ldots \ldots \ldots \ldots$} & 54.9 & 64.5 & $25 I .7$ & 190.2 \\
\hline & 54.2 & 64.7 & 258.0 & $\cdots$ \\
\hline \multirow{2}{*}{$B_{1}$, soluble in ether } & 42.2 & 66.5 & 237.2 & 171.4 \\
\hline & $42 . I$ & 67.0 & 239.1 & $\cdots$ \\
\hline \multirow{2}{*}{$C_{1}$, soluble in ether $\ldots \ldots \ldots \ldots$} & 25.4 & 98.2 & 330.0 & 230.4 \\
\hline & 25.4 & IOI.I & $\cdots$ & $\cdots$ \\
\hline \multirow{2}{*}{$A_{2}$, insoluble in ether $\ldots \ldots \ldots \ldots$} & $\{32.5$ & $\cdots$ & $\cdots$ & $\cdots$ \\
\hline & 234.8 & $\cdots$ & $\cdots$ & $\cdots$ \\
\hline \multirow{2}{*}{$\mathrm{B}_{2}, \mathrm{CHCl}_{3}$ extract of ether-insol. } & 32.2 & $7 \mathrm{~T} .2\}_{1}$ & $32 I .1$ & 243.3 \\
\hline & 32.9 & $85.7)$ & 322.3 & $\cdots$ \\
\hline \multirow{2}{*}{$\mathrm{C}_{2}$, insoluble in ether $\ldots \ldots \ldots \ldots$} & 24.2 & $\cdots$ & $\cdots \cdot$ & $\cdots$ \\
\hline & $\begin{array}{l}24.7 \\
28.6\end{array}$ & $\cdots$ & $\cdots$ & $\cdots$ \\
\hline $\mathrm{B}_{3}$, insoluble in ether or $\mathrm{CHCl}_{3}$. & $\left\{\begin{array}{l}20.0 \\
29.1\end{array}\right.$ & $\ldots$ & $\ldots$ & $\ldots$. \\
\hline \multirow{2}{*}{ Oil not precipitated from ligroin } & 65.0 & 21.6 & I93.I & I 71.4 \\
\hline & 66.0 & 21.7 & 193.9 & $\cdots$ \\
\hline \multirow{2}{*}{ Acids from ether-soluble. } & 51.8 & $23 \mathrm{I} .2$ & 268.9 & $37 \cdot 7$ \\
\hline & 52.9 & $23 I .5$ & 269.6 & $\cdots$ \\
\hline \multirow[t]{2}{*}{ Acids from ether-insoluble. } & $\{43.4$ & 265.2 & 307.0 & 42.4 \\
\hline & l 45.9 & 265.8 & 309.0 & $\cdots$ \\
\hline
\end{tabular}

The ether value was obtained by taking the difference between the mean of the saponification values and the mean of the acid values.

From the free fatty acids of $A_{1}, B_{1}$ and $C_{1}$ white crystalline products wereobtained. Crystalline products of a similar nature were abtained from the ether-insoluble portion. To show that this product did not come from any of the original oil carried down mechanically by the oxidation product, 25 grams of the oxidized oil were heated with ether, which rendered the product very brittle, and it was then finely ground. This was then extracted with ether in a Soxhlet extractor for ten hours, which removed all of the original oil and also all of the oxidized part soluble in ether. When

1 These igures do not represent the true acid value, as we found it impossible to bring the last traces of this product into solution, before titrating with potassium hydroxide. 
the product was dried after the extraction, it was of the consistency of cornmeal, and absolutely free from sticky material. It required not over fifteen minutes for complete saponification with a 25 per cent. potassium hydroxide solution; sufficient heat was generated by the reaction itself to effect the result. The fatty acids were thrown out with sulphuric acid and the solution cooled with ice so the acids could be lifted out in a lump with a stirring rod; without cooling, the acids could not be separated from the water by mechanical means. They were then thoroughly washed with water and dried; the weight was 15.2 grams. After mixing with quartz sand, the acids were extracted with ligroin. The weight of product extracted was 1.2 grams. This was perfectly white and was crystallized from glacial acetic acid. The part insoluble in ligroin was then extracted with ether. The ether extract weighed Io grams; that insoluble in ether weighed nearly 4 grams and was very dark in color.

By fractional crystallization, I.2 grams of the white crystalline product were separated into two products melting at $60^{\circ}-6 \mathrm{r}^{\circ}$ and $52^{\circ}-53^{\circ}$. This required a large number of fractions to be made, and at the end of this work but very small amounts of the two products were obtained. We are disposed to think that these two products are respectively palmitic and myristic acids, although it is difficult to understand how these acids could have been present in the product from which they were obtained, for, as pointed out above, this product had been extracted for ten hours with ether.

The fact that the benzene solution of linseed oil could not be brought to have a lower iodine number than $3 \mathrm{I}$ by continued aspiration of air through the solution, shows conclusively that the products of which linseed oil is composed can not be completely oxidized by air. From what is known of oleic acid and its properties, there is no reason to doubt but that the iodine number was produced, at least in part, from the unchanged oleic acid or olein. This, however, is but in agreement with the well-known fact that oleic acid is practically unacted upon by the oxygen of the air. From the large increase in the acid value, it is evident that the oxidation of linseed oil by air takes place to a considerable extent through the oxidation of the glycerin of the glycerides. While we have been unable, for lack of time, to attempt. an isolation of the product having the sharp, characteristic odor of acrolein, yet 
it is not impossible that acrolein may be formed during this progress of oxidation. That the air oxidizes linseed oil with the formation of carbon dioxide, formic and acetic acids, has been shown by Mulder ${ }^{1}$, from which we can see that the chemical changes taking place during the drying of the glycerides are complex. That the acrolein-like odor came from the linseed oil and not from the ligroin, was determined by a blank experiment. It is also evident that the oxidation in ligroin by means of air gives rise to products that are still unsaturated. While no acetyl values were determined, yet it was sufficiently well shown by Hazura $^{2}$ that products containing the hydroxyl group are formed. That we were, for example, dealing with a product free from any unoxidized oil or free oleic acid, let us consider such a product as $\mathrm{B}_{2}$ or $\mathrm{C}_{2}$. If any unoxidized oil was carried down mechanically with the oxidized product, it must have been in small quantities. If any such occluded oil or free oleic acid were present, it must have been completely removed by the subsequent maceration and washing of the product with ligroin, and in the long and thorough extraction with ether. Even after such treatment, the iodine number of $\mathrm{C}_{2}$ was found to be 24.5 (mean value), while in the case of $B_{2}$ it was 33.6 (mean value), and in $B_{3}$ it was 28.9 (mean value). From this we must conclude that the oxidized product thrown out from a ligroin solution is an unsaturated hydroxylated product.

What the exact chemical nature of these products is, it is, at present, impossible to say; nor can we, as yet, be assured that the product obtained from ligroin is identical with the product obtained by oxidation without a solvent. Bauer and Hazura ${ }^{3}$ obtained, by Hübl's method, an iodine number of 28.8 and 29.4 for the free acids obtained from linoxyn. We obtained an iodine number of 43.4 and 45.9 from our corresponding product, $i$. $e$, the free acids of the ether-insoluble portion.

The ether values 37.7 and 42.4 , obtained from the acids of the ether-soluble and ether-insoluble products, point to the presence of lactones in the acids, and it is altogether probable that lactones are also present in the other products given in the table, and that the ether values do not represent alone the amount of glycerin in

1 "Chemie der Austrocknenden Oele." p. rog.

${ }^{2}$ Monatsh. Chem, 9,462 and 463 (1888).

s Ibid., p. 467. 
these unsaponified products, but also the lactones present. Lekowitsch," in an article "On Oxidized Oils," points out the probable presence of lactones in solidified linseed oil (linoleum mass) through the difference in the acid and saponification values from the free acids.

The question has arisen whether or not the product with which we worked, which was insoluble in ether, was identical with Mulder's linoxyn. So far as characteristics are concerned, our ether-insoluble product was identical, except that Mulder says that linoxyn is insoluble in alcohol, while we have found that our product dissolved after long-continued boiling with a large excess of alcohol." In this, too, our results are at slight variance with Mulder's, so it may be that our product is not identical with Mulder's linoxyn, but it is more than likely that Mulder did not subject his linoxyn to as vigorous a solubility test as we have our product. Reference has already been made to our experiment regarding the solubility of our product (ether-insoluble) in chloroform and alcohol.

The oxidation product which separates from the ligroin gives a larger amount of ether-soluble product if it is extracted at once than if the product is allowed to stand for some days before extraction. This may be due to a mechanical change in the oxidation product. When first separated, the product is less tenacious and more readily permeated by the solvent, than when it has, by exposure to the air, lost all of the adhering ligroin by evaporation.

Noerdinger ${ }^{3}$ has shown that linseed oil varnish will liberate iodine from potassium iodide and has ascribed this liberation to the presence of hydrogen peroxide. We have found that our oxidation product also, when in acetic acid solution, will liberate more iodine than is liberated from a blank test. The question arises whether or not this liberation of iodine from potassium iodide is due to hydrogen peroxide. This we must answer in the negative, for we have been unable to obtain any test for hydrogen peroxide, either with chromic acid or titanic acid. The liberation of the iodine is due to the presence of an organic peroxide.

In conclusion, it should be pointed out that the question of the solubility of the oxidation product (apart from its solubility in glacial acetic acid) was doubtless more or less affected by the presence of the lead and manganese of the drier.

1 Analyst, 27, 139 (1902).

2 Except in the case of $\mathrm{B}_{2}$ as has already been noted.

3 Pharm. Centrbl., 35, 730 (1894). 